\title{
A NEW PROPERTY EQUIVALENT TO LEBESGUE INTEGRABILITY
}

\author{
ARLO W. SCHURLE
}

\begin{abstract}
Using the generalized Riemann approach to Lebesgue integration we define a new property which is equivalent to Lebesgue integrability for measurable functions. Roughly speaking, this property says that Riemann sums for sufficiently fine partitions of sufficiently small intervals can always be made arbitrarily small. We formulate this property in such a way that it applies to either Lebesgue integration or Perron integration, thus correcting a defect in earlier versions of this idea. The condition of measurability is used only in preliminary results to insure that the support of functions can always be assumed to be $G_{\delta}$-sets.
\end{abstract}

Conventions and Definitions. We take the generalized Riemann approach to integration which originated with ideas of Henstock, Kurzweil, and McShane. The reader is referred to McLeod [1] and Pfeffer [3] for more details than we provide here. The author is grateful to W. Pfeffer for introducing him to this approach and to the problems which led to the results given here as well as to the University of Petroleum and Minerals for its support of this research.

Throughout the paper we fix a real interval $I=[a, b]$ and a nondecreasing function $\alpha$ on $I$. Since we will be interested only in integrability of functions on $I$, we extend $\alpha$ outside $I$ by letting $\alpha(x)$ be $\alpha(a)$ for $x<a$, and $\alpha(b)$ for $x>b$. In order to avoid complications caused by the endpoints of $I$ we stipulate that the interior of an interval $J$ means its topological interior with respect to $I$.

As usual, the function $\alpha$ induces a Borel measure on the real numbers after we set $\alpha([r, s])=\alpha(s)-\alpha(r)$. Since $\alpha$ may not be continuous we have need for the function $\alpha^{\prime}$ defined by $\alpha^{\prime}(x)=\lim _{t \rightarrow 0} \alpha([x-t, x+t])$.

Suppose that $J$ is a subinterval of $I$. By a partition $P$ of $J$ we mean a collection $\left\{I_{1}, I_{2}, \ldots, I_{n} ; x_{1}, x_{2}, \ldots, x_{n}\right\}$, where $I_{1}, I_{2}, \ldots, I_{n}$ are nonoverlapping (their interiors are pairwise disjoint) intervals whose union is $J$, and each $x_{k}$ is a point of $J$. We say that the $x_{k}$ 's are points of $P$ and that $x_{k}$ is the point of $P$ corresponding to $I_{k}$. If we require that each point of $P$ belongs to its corresponding interval, then we say that $P$ is a Perron partition.

If $\delta$ is a positive function on the interval $I$ then we say that a partition $P$ as given above is $\delta$-fine if $I_{k}$ is contained in $\left(x_{k}-\delta\left(x_{k}\right), x_{k}+\delta\left(x_{k}\right)\right)$ for each $k$. We should note that for any positive function $\delta$ there are $\delta$-fine partitions such that each point is in the interior of its corresponding interval. See [3, p. 4].

Received by the editors January 22, 1985. Presented to the Society, August 13, 1985, Laramie, Wyoming.

1980 Mathematics Subject Classification. Primary 26A39, 26 A42.

Key words and phrases. Lebesgue integration, generalized Riemann integration, Perron integration.

(C) 1986 American Mathematical Society $0002-9939 / 86 \$ 1.00+\$ .25$ per page 
Let $f$ be any function on the subinterval $J$ of $I$ and let $P$ be a partition of $J$, say

$$
P=\left\{I_{1}, I_{2}, \ldots, I_{n} ; x_{1}, x_{2}, \ldots, x_{n}\right\} .
$$

We denote the sum $\sum_{k=1}^{n} f\left(x_{k}\right) \alpha\left(I_{k}\right)$ by $\sigma(f, P, \alpha)$ or just $\sigma(f, P)$ when no confusion results. If there is a number $A$ such that for each positive $\varepsilon$ there is a positive function $\delta$ on $J$ for which $|\sigma(f, P)-A|<\varepsilon$ whenever $P$ is a $\delta$-fine partition of $J$, then we say that $f$ is Lebesgue-Stieltjes integrable (or just $L$-integrable) with respect to $\alpha$ on $J$. If we only require that $\sigma(f, P)$ is near $A$ for $P$ a $\delta$-fine Perron partition of $J$, then we say that $f$ is Perron-Stieltjes integrable (or just $P$-integrable) with respect to $\alpha$ on $J$. We denote $A$ by $L \int f d \alpha$ in the Lebesgue case and by $P \int f d \alpha$ in the Perron case. This definition of Lebesgue-Stieltjes integral does not agree exactly with that in [4] but does agree with [3].

We now define four closely related properties which we call Property LSRS, Property LSRS', Property LSRS-P and Property LSRS'-P. These acronyms come from Locally Small Riemann Sums and Locally Small Riemann Sums-Perron. We say that a function $f$ on $I$ has Property LSRS on $I$ with respect to $\alpha$ if for each $b$ in $I$, and for each positive number $\varepsilon$, there is a positive function $\delta$ on $I$ for which $\left|\sigma(f, P)-f(b) \alpha^{\prime}(b)\right|<\varepsilon$ whenever $P$ is a $\delta$-fine partition of an interval containing $b$ in its interior and lying in $(b-\delta(b), b+\delta(b))$. Property LSRS-P is the same except that the condition need hold only for Perron partitions $P$. Note that the function $\delta$ may depend on both $b$ and $\alpha$.

We say that a function $f$ on $I$ has Property LSRS' with respect to $\alpha$ on $I$ if for each $b$ in $I$, and for each positive number $\varepsilon$, there is a positive function $\delta$ on $I$ for which $|\sigma(f, P)|<\varepsilon$ whenever $P$ is a $\delta$-fine partition of an interval lying in $(b-\delta(b), b+\delta(b))-\{b\}$. Finally, Property LSRS'-P is the same except that, again, the condition need hold only for Perron partitions $P$.

Preliminary Results. Property LSRS'-P first appeared in [5], where an example is given to show that Property LSRS' does not imply Lebesgue integrability. However, the same paper provides a proof of the following theorem, which is fundamental to the results we prove here.

THEOREM 1. An $\alpha$-measurable function $f$ on the interval $I$ is Perron-Stieltjes integrable on I with respect to $\alpha$ if and only if $f$ has Property $L S R S^{\prime}-P$ with respect to $\alpha$ on $I$.

The next result not only shows that our new formulation of the locally small Riemann sum property is actually the same as the previous one for the Perron case, but it also allows us to make use of Theorem 1 in the proof for the Lebesgue case. The verification of Theorem 2 is not difficult and we omit it. Theorem 3 is then an immediate corollary of Theorems 1 and 2.

THEOREM 2. Properties LSRS-P and LSRS'-P are equivalent.

TheOREM 3. Property LSRS-P is equivalent to Perron-Stieltjes integrability for $\alpha$-measurable functions. 
We need one more elementary result concerning these properties. The proof is not difficult but we present it here for completeness.

THEOREM 4. Suppose that the function $f$ has Property LSRS with respect to $\alpha$ on I and that $b$ is a point of $I$. Let the function $\hat{f}$ be defined by letting $\hat{f}$ agree with $f$ except at $b$ and by setting $\hat{f}(b)=0$. Then $\hat{f}$ also has Property LSRS with respect to $\alpha$ on $I$.

Proof. Certainly the only problem is at point $b$. Let $\varepsilon$ be a positive number. There is a positive function $\delta^{\prime}$ on $I$ which satisfies the condition imposed by Property LSRS for $f$ and $\varepsilon / 2$ at $b$, and for which

$$
|f(b)|\left(\alpha\left(\left[b-\delta^{\prime}(b), b+\delta^{\prime}(b)\right]\right)-\alpha^{\prime}(b)\right)<\varepsilon / 2 .
$$

Define the positive function $\delta$ by letting $\delta(b)=\delta^{\prime}(b)$, and $\delta(x)$ be the minimum of $\delta^{\prime}(x)$ and $|x-b|$ when $x \neq b$.

Now let $P$ be a $\delta$-fine partition of an interval which contains $b$ in its interior and which lies in $(b-\delta(b), b+\delta(b))$. The definition of $\delta$ forces the interval $I_{b}$ of $P$, which contains $b$, to have $b$ as its corresponding point. Using the definition of $\hat{f}$ and properties of $\delta^{\prime}$ we have

$$
\begin{aligned}
\left|\sigma(\hat{f}, P)-\hat{f}(b) \alpha^{\prime}(b)\right| & =\left|\sigma(\hat{f}, P)+f(b) \alpha\left(I_{b}\right)-f(b) \alpha\left(I_{b}\right)\right| \\
& =\left|\sigma(f, P)-f(b) \alpha\left(I_{b}\right)\right| \\
& \leqslant\left|\sigma(f, P)-f(b) \alpha^{\prime}(b)\right|+|f(b)|\left(\alpha\left(I_{b}\right)-\alpha^{\prime}(b)\right) \\
& <\varepsilon / 2+\varepsilon / 2=\varepsilon .
\end{aligned}
$$

Thus, $\hat{f}$ has Property LSRS.

The Main Result. Theorem 5 now completes the promise of the abstract and, together with Theorem 3, shows that Property LSRS is the correct formulation of locally small Riemann sums.

THEOREM 5. An $\alpha$-measurable function $f$ on the interval $I$ is Lebesgue-Stieltjes integrable on I with respect to $\alpha$ if and only if $f$ has Property LSRS with respect to $\alpha$ on I.

Proof. The fact that a Lebesgue-Stieltjes integrable function $f$ has Property LSRS comes essentially from the continuity of the integral. The proof in detail is exactly analogous to that given for Property LSRS'-P in [5], so we omit it here.

Suppose that the function $f$ has Property LSRS with respect to $\alpha$ on $I$. Recall that $f$ is Lebesgue-Stieltjes integrable if and only if each of the functions $f^{+}=$ $(|f|+f) / 2$ and $f^{-}=(|f|-f) / 2$ are Lebesgue-Stieltjes integrable. Furthermore, a nonnegative function is Lebesgue-Stieltjes integrable if and only if it is Perron-Stieltjes integrable [3, p. 46]. Thus, by Theorem 3 we will be done if we show that $f^{+}$and $f^{-}$ both have Property LSRS-P. We will do this for $f^{+}$, the other case being exactly the same. 
Let $b$ be any point of $I$ and let $\varepsilon$ be a positive number. Suppose that $f(b) \geqslant 0$. There is a positive function $\delta$ on $I$ such that if $P$ is a $\delta$-fine partition of an interval containing $b$ in its interior and lying in $(b-\delta(b), b+\delta(b))$, then $\mid \sigma(f, P)-$ $f(b) \alpha^{\prime}(b) \mid<\varepsilon / 2$ and

$$
f(b)\left(\alpha([b-\delta(b), b+\delta(b)])-\alpha^{\prime}(b)\right)<\varepsilon / 2 .
$$

Let $P$ be a $\delta$-fine Perron partition of an interval containing $b$ in its interior and lying in $(b-\delta(b), b+\delta(b))$. If $x_{k}$ is a point of $P$ for which $f\left(x_{k}\right)<0$, then replace $x_{k}$ by $b$ in the partition. We then get a new partition $Q$ of the same interval. $Q$ is no longer a Perron partition but now $\sigma\left(f^{+}, Q\right)=\sigma(f, Q)$. We also have

$$
\begin{aligned}
0 & \leqslant \sigma\left(f^{+}, Q\right)-\sigma\left(f^{+}, P\right) \\
& \leqslant f(b)\left(\alpha([b-\delta(b), b+\delta(b)])-\alpha^{\prime}(b)\right)<\varepsilon / 2 .
\end{aligned}
$$

Finally,

$$
\left|\sigma\left(f^{+}, P\right)-f^{+}(b) \alpha^{\prime}(b)\right| \leqslant\left|\sigma\left(f^{+}, P\right)-\sigma\left(f^{+}, Q\right)\right|+\left|\sigma(f, Q)-f(b) \alpha^{\prime}(b)\right|<\varepsilon .
$$

Thus, $f^{+}$has Property LSRS-P at points $b$ for which $f(b) \geqslant 0$.

Now suppose that $f(b)<0$. Consider the function $\hat{f}$ which agrees with $f$ except that $\hat{f}(b)=0$. By Theorem 4 this function still has Property LSRS. The above argument shows that the function $\hat{f}^{+}$has Property LSRS-P at the point $b$. But $\hat{f}^{+}=f^{+}$. We are done.

\section{REFERENCES}

1. R. M. McLeod, The generalized Riemann integral, Carus Math. Monographs, vol. 20, Math. Assoc. America, Washington D.C., 1980.

2. I. P. Natanson, Theory of functions of a real variable. II, transl. by L. F. Boron, Frederick Ungar, New York, 1980.

3. W. F. Pfeffer, The Riemann-Stieltjes approach to integration, Technical Report No. 187, National Research Institute for Mathematical Sciences, Pretoria, South Africa.

4. S. Saks, Theory of the integral, 2nd revised ed., transl. by L. C. Young, Dover, New York, 1964.

5. A. Schurle, A function is Perron integrable if it has locally small Riemann sums, J. Austral. Math. Soc. (to appear).

Department of Mathematical Sciences, University of Petroleum and Minerals, Dhahran 31261, Saudi Arabia 